\title{
ASSESSING COMPLEX DAMAGE USING PRE-DISASTER OPTICAL AND POST- DISASTER POLSAR DATA
}

\author{
Minyoung Jung ${ }^{1}$, Minkyung Chung ${ }^{1}$, Yongil Kim ${ }^{1, *}$ \\ ${ }^{1}$ Dept. of Civil and Environmental Engineering, Seoul National University, 1 Gwanak-ro, Gwanak-gu, Seoul 08826, Korea - \\ (worldhurrah, mkjung4876, yik)@snu.ac.kr
}

Commission III, ICWG III/IVa

KEY WORDS: Damage Assessment, Data Fusion, VHR Optical Data, PolSAR Data, Tsunami

\begin{abstract}
:
Combining pre-disaster optical and post-disaster synthetic aperture radar (SAR) data is regarded as desirable for timely damage assessment, which is essential for the prompt rescue operation. Due to the extreme differences between the two data, however, this combination has not been practically used in the previous research. In this paper, a method to assess the various types of damage caused by disasters using the desirable data combination, particularly pre-disaster very high resolution optical data and post-disaster polarimetric SAR data. The proposed method is a rule-based classification, and uses diverse components derived from the two data such as normalized difference vegetation index, polarization orientation angle, SPAN, and entropy. The proposed method was applied to the case study of the 2011 tsunami in Japan. The experimental results demonstrated the potential of the proposed method to assesses the types of tsunami-induced damage in urban and vegetated areas. The achievement in this paper is expected to facilitate efficient and fast disaster-induced complex damage assessment.
\end{abstract}

\section{INTRODUCTION}

The use of satellite imagery has emerged as a valuable source of information for rapid rescue operation because disasterlocations are usually not accessible (Gueguen and Hamid, 2015) Numerous methods have been developed to assess disasterinduced damage by employing satellite images. Most of them were based on the change detection approach, which compares the post-disaster satellite imagery to the pre-disaster satellite imagery and considers the changes between them as damages. The previous methods tend to use the similar types of the pre and the post-disaster images such as both images acquired by VHR optical sensors (Gueguen and Hamid, 2015; Mansouri and Hamednia, 2015) or both by SAR sensors (Arciniegas et al., 2007; Chen and Sato, 2013; Park et al., 2013). Relatively fewer methods (Brunner et al., 2015; Plank et al., 2016) fused the different types of images. It is because that the inherent differences from the different radiometric and geometric characteristics between sensors are incorrectly considered as damage induced by disasters, and it is, therefore, difficult to accurately assess the damage.

The method fusing the different types of images is essential in terms of the achieving timely damage assessment for the prompt rescue operation, because the immediate acquisition of the postdisaster image, of which type is similar to the pre-disaster image is practically difficult. Particularly, an optical sensor, which is sensitive to the weather and illumination conditions, is limited to be operated immediately after the disasters occur. On the contrary, a synthetic aperture radar (SAR) is appropriate for disaster sites due to its relative insensitivity to those conditions. However, less abundant pre-disaster SAR images exist. In this respect, the pre-disaster optical data and the post-disaster SAR data are regarded as a desirable data combination for rapid damage assessment (Brunner et al., 2015).
Recent disasters usually occur over vast areas and result in complex damage in various land cover types. The complex damage can be efficiently detected by using fully polarimetric SAR (PolSAR) data since it provides sufficient information of ground objects and allows more useful results with its additional polarization scattering mechanism compared to a single polarization mode. Consequently, there has been increasing research employing PolSAR for damage investigation (Chen and Sato, 2013; Park et al., 2013; Plank et al., 2016).

This paper is aimed at developing a novel method to assess complex damage using the desirable data combination, in particular, the pre-disaster VHR optical data and the postdisaster PolSAR data. Damage assessment using the two data is a challenging task due to their extreme radiometric and geometric differences. A rule-based classification is established in this paper to investigate the complex damage. The proposed method can detect three types of the damage: (1) Physically destroyed buildings, (2) Inundated croplands, (3) Destroyed windbreak forest. In order to achieve the efficient damage investigation result, the proposed method uses various components derived from optical and PolSAR data such as normalized difference vegetation index (NDVI), polarization orientation angle (POA), SPAN, and entropy.

The rest of this paper is organized as follows. In Section 2, we define the damage types in the complex damage, particularly induced by tsunamis. The rule-based classification for damage assessment of each type is described in detail in Section 3. In Section 4, we evaluate the performance of the proposed method using KOMPSAT-2 data and ALOS/PALSAR-1 data acquired before and after the 2011 tsunami in Japan, respectively. Finally, we conclude the paper in Section 5.

\footnotetext{
* Corresponding author
} 


\section{COMPLEX DAMAGE CAUSED BY TSUNAMI}

The differences between the pre-disaster VHR optical data and the post-disaster fully PolSAR data can be classified in terms of the causes such as radiometric and geometric differences between the sensors, seasonal differences usually appearing in vegetated areas, and disaster-induced differences. Therefore, the proposed method in this paper should be able to distinguish the disaster-induced differences from others. There is no definitive comparison method to define all kinds of differences between optical and PolSAR data. Furthermore, each polarimetric parameters derived from the PolSAR data is specialized to monitor different ground objects. Therefore, the complex damage investigation is individually designed according to the damage types.

A tsunami focused in this paper causes two typical types of damage, inundation and destruction. Since the restoration of tsunami damage is dissimilar according to land covers, we analysis the two typical types of damage in terms of land cover types.

Detecting inundated areas is a relatively straightforward task for detecting destroyed areas since the water bodies are normally distinguishable in both optical and SAR images. The backscattering signals of water surfaces is lower than those of other land covers, and numerous methods have been developed to detect water bodies from the two images (Nath and Deb, 2010; Hong et al., 2015). By using such methods, the simplest way to detect inundated areas is to compare the detected water bodies derived from the two data.

The torrent of water destroys diverse ground objects in the catastrophic site. Generally, destruction in urban areas is directly related to human casualties. In this respect, we investigate the destroyed buildings in detail. Furthermore, a windbreak forest located along a seashore, which is another object possibly destroyed by the tsunami water, is also investigated.

\section{METHODOLOGY}

To directly compare between optical and SAR data, the methods based on simulation have been proposed (Wang and Jin, 2012; Brunner et al., 2015). Although the sufficient number of the polarimetric parameters of PolSAR data, few parameters are comparable with the parameters possibly obtained from the optical data. Therefore, a rule-based classification is alternatively adopted to assess the complex damage by the tsunami.

\subsection{The components derived from the data}

3.1.1 Components of the VHR optical data: Before describing the rule-based classification in detail, the components in this paper are briefly introduced. Two components are derived from the VHR optical data, an NDVI and a POA. The former is a well-known index and has been widely used to monitor the condition of the vegetation and to classify the land covers. The actual values of the NDVI should be obtained after the radiometric calibration; however, the radiometric calibration is quite complicated and does not critically affect the damage assessment results. Therefore, the NDVI is calculated using digital number (DN) of the nearinfrared (NIR) and red bands of the VHR optical data without any radiometric calibration.

$$
\mathrm{NDVI}=\frac{\mathrm{DN}_{\mathrm{NIR}}-\mathrm{DN}_{\mathrm{Red}}}{\mathrm{DN}_{\mathrm{NIR}}+\mathrm{DN}_{\mathrm{Red}}}
$$

Because the POA is the unique component of PolSAR data, we simulated it from VHR optical data by the method in Jung et al. (2018). They proposed the entire process of simulation POAs using VHR optical data with lines. The POA is originally related to the slopes of a terrain and radar look angles, and the walls of buildings affect the POAs in urban areas. Therefore, the POA in urban areas can be calculated once the building orientation angle defined.

3.1.2 Components of the PolSAR data: PolSAR data is originally represented using the scattering matrix $\mathbf{S}$ (Lee et al., 2000). The coherency matrix $\mathbf{T}$, which derived from $\mathbf{S}$ based on the reciprocity condition $\left(\mathrm{S}_{\mathrm{hv}} \approx \mathrm{S}_{\mathrm{vh}}\right)$, is defined as:

$$
\mathbf{T}=\left\langle k k^{* T}\right\rangle=\left[\begin{array}{lll}
T_{11} & T_{12} & T_{13} \\
T_{21} & T_{22} & T_{23} \\
T_{31} & T_{32} & T_{33}
\end{array}\right]
$$

where $\langle\cdot\rangle$ indicates averaging and $k$ is a Pauli scattering vector. The superscripts $*$ and ${ }^{T}$ denote the complex conjugate and the transpose, respectively. By using the component of $\mathbf{T}$, the used component derived from the PolSAR data can be calculated. A SPAN image represents the total scattering power of PolSAR data as Equation (3).

$$
\mathrm{SPAN}=T_{11}+T_{22}+T_{33}
$$

The POA $\theta$ is calculated as follows (Lee et al., 2000):

$$
\theta=\frac{1}{4} \tan ^{-1}\left(\frac{2 \operatorname{Re}\left(T_{23}\right)}{T_{22}-T_{33}} \pm n \pi\right)
$$

where $\operatorname{Re}\left(T_{23}\right)$ is the real part of $T_{23}$ and $\pm n \pi(n=0,1)$ is necessary to prevent the coherency matrix from being rotated toward the wrong axis (Chen et al., 2013).

An entropy $(H)$ is a useful descriptor of the randomness of the backscattering signals. If the entropy is low, one dominant scattering exists among the surface, double-bounce, and volume scattering. The maximum value of entropy is 1 and indicates that the backscattering is totally random.

$$
H=-\sum_{k=1}^{3} P_{k} \log _{3}\left(P_{k}\right)
$$

where $P_{i}=\lambda_{i} / \sum_{k=1}^{3} \lambda_{i}$ and $\lambda_{i}$ are the eigenvalues of $\mathbf{T}$ (Lee and Pottier, 2009).

\subsection{Method to assess the complex damage}

To assess the complex damage, we first extract the candidate areas of three land covers-vegetated areas, water surfaces, and forests. In consideration of the reflectance characteristic of each land cover, the candidates are classified with the Otsu threshold method (Otsu, 1979). Table 1 shows the rules of the classification of three land covers.

With the extracted candidate areas using these rules, the damaged areas are extracted. The inundated croplands are the areas which were the vegetated candidates in the pre-disaster optical image and became the water surfaces in post-disaster 


\begin{tabular}{ccl} 
Land cover & \multicolumn{1}{c}{ Rule } & \multicolumn{1}{c}{ Reflectance characteristic } \\
\hline Vegetation & NDVI $\geq$ threshold & $\begin{array}{l}\text { Vegetated areas such as croplands, pastures, and forests, have higher values of the } \\
\text { NDVI than those of other land covers. } \\
\text { Water surfaces without winds have low brightness values in both optical data and SAR } \\
\text { data } \\
\text { The backscattering signals of forests are the mixtures of various scattering mechanism } \\
\text { reflected from numerous objects including grounds, trunks, branches, etc. } \\
\text { Consequently, the randomness of the backscattering from the forests necessarily } \\
\text { increases and results in high value of the entropy. }\end{array}$
\end{tabular}

Table 1. Classification rule of each land cover. Each threshold is calculated by Otsu method.

PolSAR image. The other inundated land cover can be defined similarly. The damaged forest is the areas with high values of the NDVI and the SPAN, but low values of the entropy. These two types of tsunami-induced damage cannot be quantified their severities because the components used in this paper do not have any quantitative relationship with each other.

Unlike the above two types of damage, the damage level of the destroyed buildings can be quantitatively determined, as shown in Chen and Sato (2013) and Jung et al. (2018). We adopt the indicator D as Eq. (6) to generate a building damage level map (Jung et al., 2018). The higher value of the D is, the more severely destroyed buildings exist.

$$
\mathrm{D}=\left\{\begin{array}{cl}
r_{\text {dir, pre }}-r_{\text {dir, post }} & \text { if } r_{\text {dir, pre }}-r_{\text {dir, post }} \geq 0 \\
0 & \text { if } r_{\text {dir }, \text { pre }}-r_{\text {dir, post }}<0
\end{array}\right.
$$

where $r_{d i r, p r e}$ and $r_{d i r, p o s t}$ are the directional dispersions of preand post-disaster POAs. Since this approach cannot avoid the errors from forests where the POAs fluctuate, the vegetated areas are eliminated from the generated building damage level map.

\section{EXPERIMENTAL RESULTS}

\subsection{Study site and data description}

The study site is located in Ishinomaki of Miyagi prefecture, reported as severely damaged by the 2011 tsunami induced by an earthquake of magnitude 9.0 (National Police Agency, 2018) As shown in Fig. 1(a), the catastrophic site is composed of various land covers, including urban, croplands, and forests.

Table 2 shows the data used for assessing the damage caused by the 2011 Japan tsunami by comparing the pre-tsunami VHR optical data and post-tsunami PolSAR data. The VHR optical data was acquired on October 1, 2009, by KOMPSAT-2 (Fig. 1(a)). The fully PolSAR data was acquired on April 8, 2011, by the ALOS/PALSAR-1 (Fig. 1(b)). Some clouds cover several areas in the KOMPSAT-2 image but do not cover the areas near the seashore, which are mainly damaged by the tsunami.

\begin{tabular}{c|cc} 
Data Type & VHR optical data & PolSAR \\
Sensor & KOMPSAT-2 & ALOS/PALSAR-1 \\
Acquired Data & October 1, 2009 & April 8, 2011 \\
Incidence Angle & - & $23.836^{\circ}$ \\
& $1 \mathrm{~m}$ & $3.43 \mathrm{~m}$ \\
Spatial & (Panchromatic) & (Azimuth) \\
Resolution & $4 \mathrm{~m}$ & $23.18 \mathrm{~m}$ \\
& (Multi-spectral) & (Ground-range)
\end{tabular}

Table 2. Description of the remote sensing data

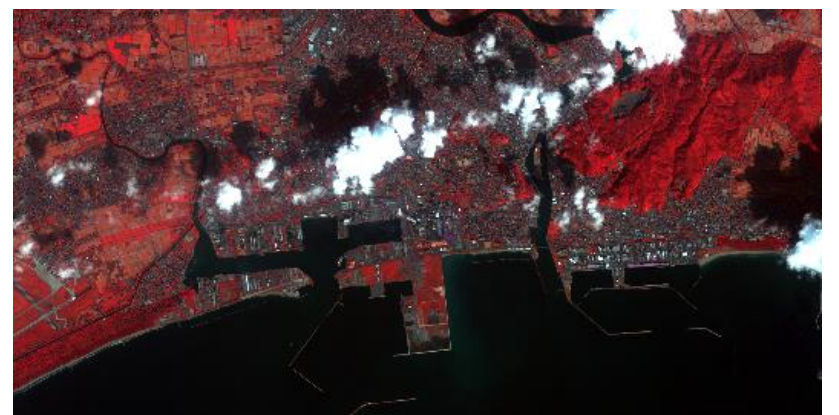

(a)

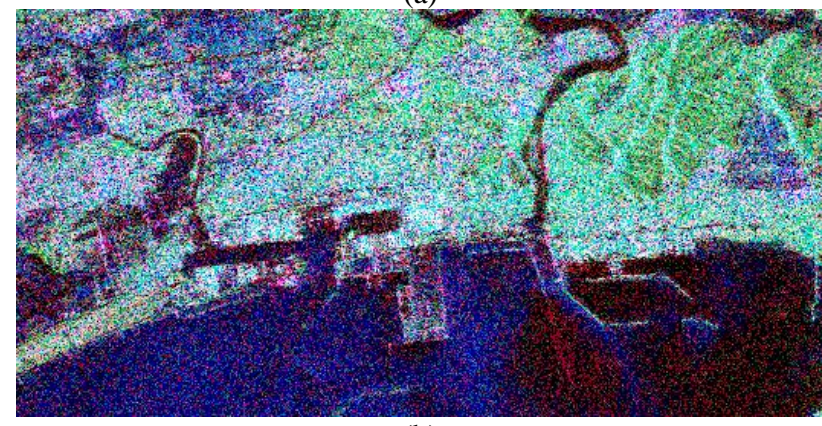

(b)

Figure 1. (a) False colour (NIR-G-B) image of the KOMPSAT2 data; (b) Pauli RGB image of the ALOS/PALSAR-1 data.

The two data were indirectly registered by the same approach in Jung et al. (2018). The single-look ALOS/PALSAR-1 data was first pre-processed. The PolSAR data was seven-multi-looked in the azimuth direction and geocoded to the map projection of KOMPSAT-2 data. Afterwards, the transformation model was calculated by the normalized mutual information (Studholme et al., 1999) between the panchromatic image of the VHR optical data and the SPAN image of the pre-processed PolSAR data, and applied to every component derived from the two data.

The performance of the proposed approach to assess physically destroyed buildings was examined using the building damage map of the 2011 Japan tsunami visually interpreted using aerial photos, as shown in Fig. 2(a) (WorldMap, 2018). Since no appropriate reference data exist in terms of the vegetated areas, we additionally referred the GoogleEarth image acquired on April 6, 2011, two days before the PolSAR data obtained. It was predicted that the inundated vegetated areas and damaged forests existed near the acquisition day of the PolSAR data (Fig. 2 (a) and (b)). However, the urban areas were not inundated. 


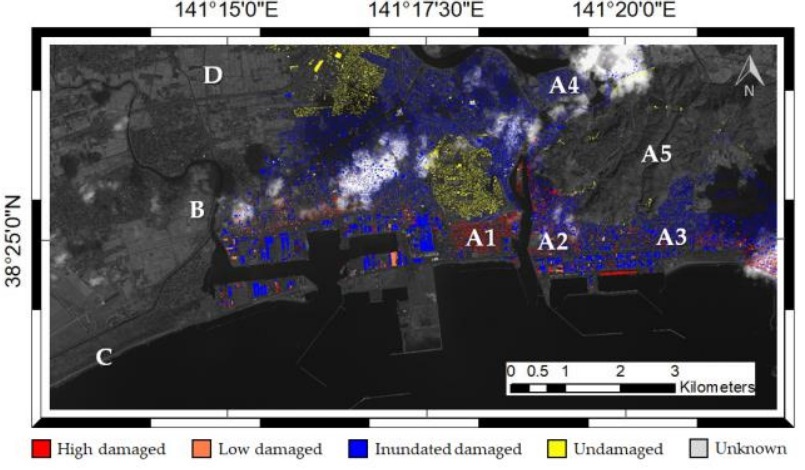

(a)

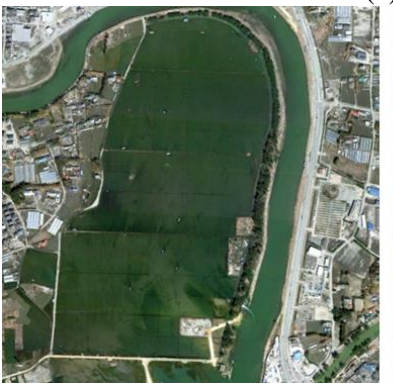

(b)

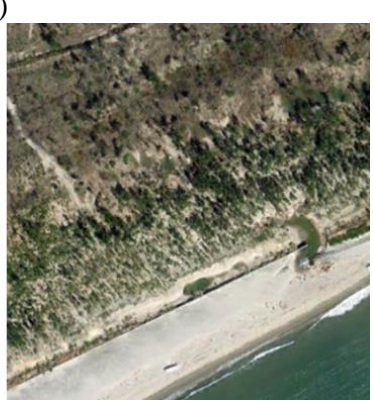

(c)
Figure 2. (a) Building damage map. The regions of interest (ROIs) for the validation are labelled as $\mathbf{A 1}$ to $\mathbf{A 5}, \mathbf{B}, \mathbf{C}$, and $\mathbf{D}$;

(b) The GoogleEarth image of the inundated cropland, $\mathbf{B}$; and

(c) The GoogleEarth image of the damaged forest (windbreak),

C.

\subsection{Investigation of the complex da mage}

4.2.1 Components derived from two data: Figure 3 shows the components derived from the KOMPSAT-2 and ALOS/PALSAR-1 data as expected in Section 3.2. It is found that the method proposed in Jung et al. (2018) well simulated POAs of the urban areas as compared to PolSAR POAs. Even though the absence of the radiometric calibration, the vegetated areas, including the croplands and forests, show the high values of the NDVI. In terms of the PolSAR data, water bodies have low values of the SPAN. The values of the entropy are high over the forest areas, of which backscattering mechanism is complicated.

4.2.2 Investigation of the vegetated areas: The rule-based classification generated the damage map of the vegetated areas, as shown in Fig. 4. Due to the absence of the appropriate reference data for the damaged vegetated areas, we visually evaluated the result with the GoogleEarth image. The actual inundated area, $\mathbf{B}$, was extracted as the inundated croplands by the proposed method. The subimage at the bottom right corner of Fig. 4 is the magnified image of the windbreak forest. It shows that the tsunami destroyed the windbreak forests. The small surface of the detected damaged windbreak forests is partly reasonable as considered that the windbreak forest in $\mathbf{C}$ was not severely damaged, as shown in Fig. 2(c).

Meanwhile, the errors in $\mathbf{D}$ demonstrated that the proposed method could not distinguish the temporal changes in the vegetated areas from the changes due to the damage. The vegetated areas were bare soil near the acquisition date of the PolSAR data because it was before the planting season. Although the vegetated areas in $\mathbf{D}$ were not inundated or destroyed at that moment, they were determined as the damaged vegetated areas. The additional information on other components possibly derived from pre-tsunami VHR optical data and post-tsunami PolSAR data can solve this limitation.

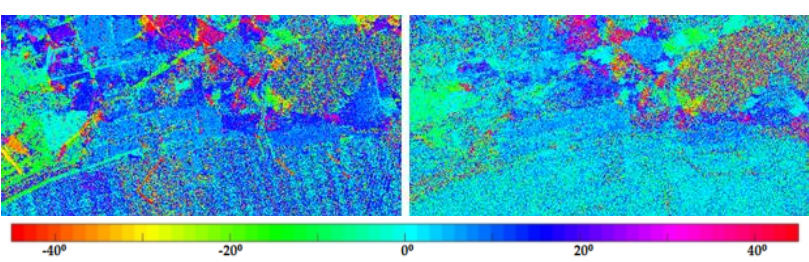

(a)

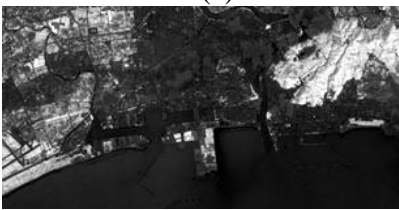

(c)

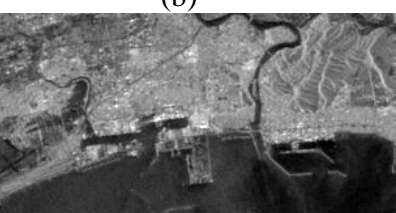

(d)

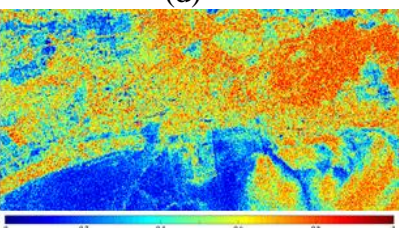

(e)
Figure 3. Derived components: (a) Simulated POAs; (b) PolSAR POAs; (c) NDVI; (d) SPAN; and (e) Entropy.

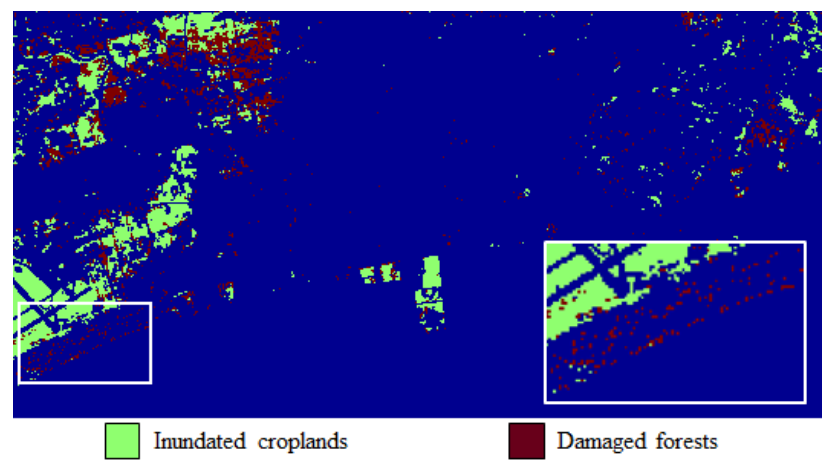

Figure 4. Damage map of the vegetated areas.

4.2.3 Investigation of the destroyed buildings: Figure 5 shows the damage level map of the destroyed buildings in the study site. The noticeable areas with the high values of the index D are the areas, where the high and low damaged buildings exist according to the reference map in Fig. 2(a).

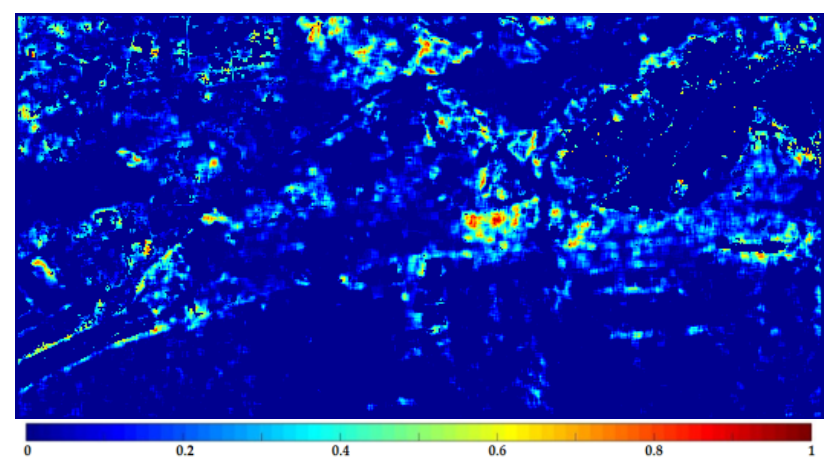

Figure 5. Damage level map of destroyed buildings

The mean values of the ROIs, $\mathbf{A 1}$ to $\mathbf{A 5}$, were calculated as Table 3 for quantitative evaluation. The mean values tend to increase as the damage level increases. It indicates that the index $\mathrm{D}$ is the useful descriptor of the severity of building 
damage. Furthermore, the forest areas, which usually have high values of the $\mathrm{D}$, are efficiently eliminated by the proposed method. Notably, the mean values of the forest in $\mathbf{A 5}$ are remarkably decreasing from 0.331 to 0.011 after eliminating process. According to the values in Table 3, the value of A5, 0.011 , is interpreted as undamaged areas.

\begin{tabular}{ccc} 
ROIs & The mean value & Damage level \\
\hline A1 & 0.442 & high \\
A2 & 0.228 & medium \\
A3 & 0.120 & low \\
A4 & 0.004 & undamaged \\
A5 & 0.013 & (forest)
\end{tabular}

Table 3. The mean values of the D index of the ROI A1 to A5.

\section{CONCLUSION}

This paper has proposed a novel method to assess the complex damage caused by tsunami based on the rule-based classification using the pre-tsunami VHR optical data and posttsunami PolSAR data. The proposed method can detect three major types of damage - the inundated croplands, damaged forest, and physically destroyed buildings by using various components derived from the two data. The experimental results demonstrated the potential of the proposed method to assesses the types of tsunami-induced damage in urban and vegetated areas. Although the proposed method is focused on the tsunami induced image, it can be applied to the damage induced by other types of disasters unless the radiometric characteristics are different. The achievement in this paper is expected to increase the availability of satellite imagery for prompt disaster-induced damage investigation.

\section{ACKNOWLEDGEMENTS}

This research was supported by a grant (2019-MOIS32-015) of Disaster-Safety Industry Promotion Program funded by Ministry of Interior and Safety (MOIS, Korea).

\section{REFERENCES}

Arciniegas, G.A., Bijker, W., Kerle, N., and Tolpekin, V.A., 2007. Coherence-and amplitude-based analysis of seismogenic damage in Bam, Iran, using ENVISAT ASAR data. IEEE Trans. Geosci. Remote Sens., 45(6), 1571-1581.

Brunner, D., Lemoine, G., and Bruzzone, L., 2015. Earthquake damage assessment of buildings using VHR optical and SAR imagery. IEEE Trans. Geosci. Remote Sens., 48(5), 2403-2420.

Chen, S.-W., Ohki, M., Shimada, M., and Sato, M., 2013. Deorientation effect investigation for model-based decomposition over oriented built-up areas. IEEE Geoscience and Remote Sensing Letters, 10(2), 273-277.

Chen, S.-W., and Sato, M., 2013. Tsunami damage investigation of built-up areas using multitemporal spaceborne full polarimetric SAR images. IEEE Trans. Geosci. Remote Sens. 50(4), 1985-1997, doi:10.1109/TGRS.2012.2210050.

Gueguen, L., and Hamid, R., 2015. Large-scale damage detection using satellite imagery, In: IEEE Conference on Comput. Vis. and Pattern Recognit., 7-12 June, 2015, Boston, MA, USA, 1321-1328.
Hong, S., Jang, H., Kim, N., and Sohn, H. G., 2015. Water area extraction using RADARSAT SAR imagery combined with landsat imagery and terrain information. Sensors, 15(3), 66526667.

Jung, M., Yeom, J., and Kim, Y., 2018. Comparison of preevent VHR optical data and post-event PolSAR data to investigate damage caused by the 2011 Japan tsunami in builtup areas. Remote Sens., 10(11), 1804.

Lee, J.S., Schuler, D.L., and Ainsworth, T.L., 2000. Polarimetric SAR data compensation for terrain azimuth slope variation. IEEE Trans. Geosci. Remote Sens., 38(5), 2153-2163.

Lee, J.S., and Pottier, E., 2009: Polarimetric Radar Imaging: From Basics to Applications. CRC Press: Boca Raton, FL, USA.

Mansouri, B., and Hamednia, Y., 2015. A soft computing method for damage mapping using VHR optical satellite imagery. IEEE J. Sel. Top. Appl. Earth Obs. Remote Sens., 8(10), 4935-4941.

Nath, R. K., and Deb, S. K., 2010. Water-body area extraction from high resolution satellite images-an introduction, review, and comparison. Int. J. Image Process. (IJIP), 3(6), 265-384.

National Police Agency. http://www.npa.go.jp/news/other/earth quake2011/pdf/higaijok yo_e.pdf (30 April 2018).

Otsu, N., 1979. A threshold selection method from gray-level histograms. IEEE Transactions on Systems, Man, and Cybernetics, 9(1), 62-66.

Park, S.-E., Yamaguchi, Y., and Kim, D., 2013. Polarimetric SAR remote sensing of the 2011 Tohoku earthquake using ALOS/PALSAR. Remote Sens. Environ., 132, 212-220.

Plank, S., Twele, A., and Martinis, S., 2016. Landslide mapping in vegetated areas using change detection based on optical and polarimetric SAR data. Remote Sens., 8(4), 307.

Studholme, C., Hill, D.L.G., and Hawkes, D.J., 1999. An overlap invariant entropy measure of 3D medical image alignment. Pattern Recognit. 32(1), 71-86.

Wang, T.-L., and Jin, Y.-Q., 2012. Postearthquake building damage assessment using multi-mutual information from preevent optical image and postevent SAR image. IEEE Geosci. Remote Sens. Lett. 9(3), 452-456

WorldMap, Ishinomaki Building Inundation. https://worldmap. harvard.edu/data/geo node:ishinomakimerge_063 (30 April 2018). 